\title{
A Note on Computational Approach to Travelling Sales Man Problem
}

\author{
Shaik. Mohiddin Shaw \\ Narasaraopeta Engineering College, \\ Narasaraopet
}

\author{
Dharmaiah Gurram \\ Narasarao PET Engineering College, \\ Narasaraopet
}

\begin{abstract}
Many real life situations for which there are no optimization algorithms which can solve polynomial time problems in the worst case. So researchers are trying for new approximation algorithms for such kinds of situations. Approximation algorithms give the solution which is close to the optimal solution of a particular situation. Traveling Salesman Problem (TSP) is a typical NP complete problem which lacks polynomial time algorithm. In this paper it is proposed an edge removal algorithm, which will give the nearly optimal solution within a limited time.
\end{abstract}

\section{General Terms}

Optimization, Travelling Sales man Problem

\section{Keywords}

Edge Removal Algorithm, Compression Algorithm, Back Tracking.

\section{INTRODUCTION}

Traveling Salesman Problem (TSP) is a famous NP hard problem and also a typical combinatorial optimization problem in Operation Research. With the increasing of number of cities, its solving time complexity grows rapidly in exponential degree, so enumerating each possible route and searching for the one with the smallest cost to optimally solve this problem becomes impossible in polynomial time. In the classical traveling salesman problem, a set of cities has to be visited in a single tour with the objective of minimizing the total length of the tour. This is one of the most studied problems in combinatorial optimization, together with its dozens of variations. In the asymmetric version of the problem, the distance from one point to another in a given space can be different from the inverse distance. This variation, known as the Asymmetric Traveling Salesman Problem (ATSP) arises in many applications; for example, one can think of a delivery vehicle traveling through one-way streets in a city, or of gasoline costs when traveling through mountain roads.

\section{THE TRAVELING SALESMAN PROBLEM}

The idea of the traveling salesman problem (TSP) is to find a tour of a given number of cities, visiting each cityexactly once and returning to the starting city where the length of this tour is minimized. TSP is of great significance in practical applications, it can be used to resolve the problems in allocation, path problem and vehicle scheduling problem and so on. The standard symmetric traveling salesman problem can be stated mathematically as follows:

Given a weighted graph $\mathrm{G}=(\mathrm{V}, \mathrm{E})$, where $\mathrm{V}$ is the set of nodes, $\mathrm{E}$ is the set of edges, and the weight $c_{i j}$ on the edge between nodes $i$ and $j$ is a non-negative value, finding the tour of all nodes that has the minimum total cost.

\section{EDGE REMOVAL ALGORITHM Algorithm:}

Step 1: Read the Adjacency Matrix, (distances between different towns).

Step 2: Apply compression algorithm on the above adjacency matrix, to remove edges and find cost tables.

Step 3: Sort the edges related to each node in ascending order.

Step 4: calculate the minimum cost array to improve the efficiency of this algorithm.

Step 5: Apply backtracking on the sorted cost tables obtained in step 3 using step 4 as an optimization step.

\subsection{Example:}

Step 1: Input:

$\begin{array}{cccccccccc}0 & 2 & 8 & 15 & 1 & 10 & 5 & 19 & 19 & 3 \\ 5 & 0 & 6 & 6 & 2 & 8 & 2 & 12 & 16 & 3 \\ 8 & 17 & 0 & 12 & 5 & 3 & 14 & 13 & 3 & 2 \\ 17 & 19 & 16 & 0 & 8 & 7 & 12 & 19 & 10 & 13 \\ 8 & 20 & 16 & 15 & 0 & 4 & 12 & 3 & 14 & 14 \\ 5 & 2 & 12 & 14 & 9 & 0 & 8 & 5 & 3 & 18 \\ 18 & 20 & 4 & 2 & 10 & 19 & 0 & 17 & 16 & 11 \\ 3 & 9 & 7 & 1 & 3 & 5 & 9 & 0 & 7 & 6 \\ 11 & 10 & 11 & 11 & 7 & 2 & 14 & 9 & 0 & 10 \\ 4 & 5 & 15 & 17 & 1 & 7 & 17 & 12 & 9 & 0\end{array}$

Step 2: Compression Step:

In this step remove these edges that are no need to visit to find the optimal solution.

By using single source shortest path algorithms it can be find the shortest paths from a node to all remaining nodes. For example, consider node number 1 , the paths from node 1 to all other nodes is like below.

Table1:

\begin{tabular}{|c|c|c|c|c|}
\hline $\begin{array}{c}\text { Source } \\
\text { Node }\end{array}$ & $\begin{array}{c}\text { Destination } \\
\text { Node }\end{array}$ & $\begin{array}{c}\text { Minimum } \\
\text { Path }\end{array}$ & $\begin{array}{c}\text { Node } \\
\text { to be } \\
\text { visited } \\
\text { first }\end{array}$ & Cost \\
\hline 1 & 2 & $(12)$ & 2 & 2 \\
\hline 1 & 3 & $(13)$ & 3 & 8 \\
\hline 1 & 4 & $(1584)$ & 5 & 5 \\
\hline
\end{tabular}




\begin{tabular}{|c|c|c|c|c|}
\hline 1 & 5 & $(15)$ & 5 & 1 \\
\hline 1 & 6 & $(156)$ & 5 & 5 \\
\hline 1 & 7 & $(127)$ & 2 & 4 \\
\hline 1 & 8 & $(158)$ & 5 & 4 \\
\hline 1 & 9 & $(1569)$ & 5 & 8 \\
\hline 1 & 10 & $(110)$ & 10 & 3 \\
\hline
\end{tabular}

Table 5: Cost table for Node 4.

\begin{tabular}{|c|c|}
\hline $\begin{array}{c}\text { Node } \\
\text { Number }\end{array}$ & Cost \\
\hline 5 & 8 \\
\hline 6 & 7 \\
\hline 9 & 10 \\
\hline
\end{tabular}

Table 6: Cost Table for Node 5.

In the next case extract the fields' node to be visited first and cost where those values in the $2^{\text {nd }}$ and $4^{\text {th }}$ column of the above table are equal. That is, nodes extracted for the above table are $2,3,5,10$.

Now the cost tables are generated for above extracted nodes and their costs.

Table 2: Cost table for node 1.

\begin{tabular}{|c|c|}
\hline $\begin{array}{c}\text { Node } \\
\text { Number }\end{array}$ & Cost \\
\hline 2 & 2 \\
\hline 3 & 8 \\
\hline 5 & 1 \\
\hline 10 & 3 \\
\hline
\end{tabular}

Cost tables for all the other nodes are given below:

Table 3: Cost Table for node 2.

\begin{tabular}{|l|l|}
\hline $\begin{array}{l}\text { Node } \\
\text { Number }\end{array}$ & Cost \\
\hline 1 & 5 \\
\hline 3 & 6 \\
\hline 5 & 2 \\
\hline 7 & 2 \\
\hline 10 & 3 \\
\hline
\end{tabular}

Table 4: Cost Table for Node 3.

\begin{tabular}{|c|c|}
\hline $\begin{array}{c}\text { Node } \\
\text { Number }\end{array}$ & Cost \\
\hline 6 & 3 \\
\hline 9 & 3 \\
\hline 10 & 2 \\
\hline
\end{tabular}

Table 7: Cost Table for Node 6 .

\begin{tabular}{|c|c|}
\hline $\begin{array}{c}\text { Node } \\
\text { Number }\end{array}$ & Cost \\
\hline 1 & 5 \\
\hline 2 & 2 \\
\hline 8 & 5 \\
\hline 9 & 3 \\
\hline
\end{tabular}

Table 8: Cost Table for Node 7

\begin{tabular}{|c|c|}
\hline $\begin{array}{c}\text { Node } \\
\text { Number }\end{array}$ & Cost \\
\hline 3 & 4 \\
\hline 4 & 2 \\
\hline
\end{tabular}

Table 9: Cost Table for Node 8.

\begin{tabular}{|c|c|}
\hline $\begin{array}{c}\text { Node } \\
\text { Number }\end{array}$ & Cost \\
\hline 1 & 3 \\
\hline 3 & 7 \\
\hline 4 & 1 \\
\hline 5 & 3 \\
\hline 6 & 5 \\
\hline 9 & 7 \\
\hline 10 & 6 \\
\hline
\end{tabular}


Table 10: Cost table for Node 9.

\begin{tabular}{|l|l|}
\hline $\begin{array}{l}\text { Node } \\
\text { Number }\end{array}$ & Cost \\
\hline 6 & 2 \\
\hline
\end{tabular}

Table 11: Cost table for Node 10.

\begin{tabular}{|c|c|}
\hline $\begin{array}{c}\text { Node } \\
\text { Number }\end{array}$ & Cost \\
\hline 1 & 4 \\
\hline 2 & 5 \\
\hline 5 & 1 \\
\hline
\end{tabular}

Step 3: Sort the above tables based on their ascending order of their costs.

Table 12: Sorted Cost table for Node 1.

\begin{tabular}{|c|c|}
\hline $\begin{array}{c}\text { Node } \\
\text { Number }\end{array}$ & Cost \\
\hline 5 & 1 \\
\hline 2 & 2 \\
\hline 10 & 3 \\
\hline 3 & 8 \\
\hline
\end{tabular}

Cost tables for all the other nodes are given below:

Table 13: Sorted Cost Table for node 2.

\begin{tabular}{|c|c|}
\hline $\begin{array}{c}\text { Node } \\
\text { Number }\end{array}$ & Cost \\
\hline 5 & 2 \\
\hline 7 & 2 \\
\hline 10 & 3 \\
\hline 1 & 5 \\
\hline 3 & 6 \\
\hline
\end{tabular}

Table 14: Sorted Cost Table for Node 3.

\begin{tabular}{|c|c|}
\hline $\begin{array}{c}\text { Node } \\
\text { Number }\end{array}$ & Cost \\
\hline 10 & 2 \\
\hline 6 & 3 \\
\hline 9 & 3 \\
\hline
\end{tabular}

Table 15: Sorted Cost table for Node 4.

\begin{tabular}{|c|c|}
\hline $\begin{array}{c}\text { Node } \\
\text { Number }\end{array}$ & Cost \\
\hline 6 & 7 \\
\hline 5 & 8 \\
\hline 9 & 10 \\
\hline
\end{tabular}

Table 16: Sorted Cost Table for Node 5.

\begin{tabular}{|c|c|}
\hline $\begin{array}{c}\text { Node } \\
\text { Number }\end{array}$ & Cost \\
\hline 8 & 3 \\
\hline 6 & 4 \\
\hline
\end{tabular}

Table 17: Sorted Cost Table for Node 6.

\begin{tabular}{|c|c|}
\hline $\begin{array}{c}\text { Node } \\
\text { Number }\end{array}$ & Cost \\
\hline 2 & 2 \\
\hline 9 & 3 \\
\hline 1 & 5 \\
\hline 8 & 5 \\
\hline
\end{tabular}

Table 18: Sorted Cost Table for Node 7

\begin{tabular}{|c|c|}
\hline $\begin{array}{c}\text { Node } \\
\text { Number }\end{array}$ & Cost \\
\hline 4 & 2 \\
\hline 3 & 4 \\
\hline
\end{tabular}

Table 19: Sorted Cost Table for Node 8.

\begin{tabular}{|c|c|}
\hline $\begin{array}{c}\text { Node } \\
\text { Number }\end{array}$ & Cost \\
\hline 4 & 1 \\
\hline 1 & 3 \\
\hline 5 & 3 \\
\hline 6 & 5 \\
\hline 10 & 6 \\
\hline 3 & 7 \\
\hline 9 & 7 \\
\hline
\end{tabular}


Table 20: Sorted Cost table for Node 9.

\begin{tabular}{|c|c|}
\hline $\begin{array}{c}\text { Node } \\
\text { Number }\end{array}$ & Cost \\
\hline 6 & 2 \\
\hline
\end{tabular}

Table 21: Sorted Cost table for Node 10.

\begin{tabular}{|c|c|}
\hline $\begin{array}{c}\text { Node } \\
\text { Number }\end{array}$ & Cost \\
\hline 5 & 1 \\
\hline 1 & 4 \\
\hline 2 & 5 \\
\hline
\end{tabular}

Step 4: Now calculate the minimum cost array

The minimum_cost_array size is equal to the number of nodes. So,

minimum_cost_array[1]

$$
=\text { Sorted_Cost_Table[1][2] }=1
$$

Minimum_Cost_Array[2]

$=$ minimum_Cost_Array[1] + Sorted_Cost_Table[2][2]

$=1+2=3$.

Minimum_Cost_Array[3]

$=$ minimum_Cost_Array[2] + Sorted_Cost_Table[3][2]

$=3+2=5$.

Minimum_Cost_Array[4]

$=$ minimum_Cost_Array[3] + Sorted_Cost_Table[4][2]

$=5+7=12$.

Minimum_Cost_Array[5]

$=$ minimum_Cost_Array[4] + Sorted_Cost_Table[5][2]

$=12+3=15$.

Minimum_Cost_Array[6]

$=$ minimum_Cost_Array[5] + Sorted_Cost_Table[6][2]

$=15+2=17$.

Minimum_Cost_Array[7]

$=$ minimum_Cost_Array[6] + Sorted_Cost_Table[7][2]

$=17+2=19$.

Minimum_Cost_Array[8]

$=$ minimum_Cost_Array[7] + Sorted_Cost_Table[8][2]

$=19+1=20$.
Minimum_Cost_Array[9]

$=$ minimum_Cost_Array[8] + Sorted_Cost_Table[9][2]

$=20+2=22$.

Minimum_Cost_Array[10]

$=$ minimum_Cost_Array[9] + Sorted_Cost_Table[10][2]

$=22+1=23$.

So, Minimum_Cost_Array[ ] =

$$
\{1,3,5,12,15,17,19,20,22,23\} .
$$

Step 5: Apply Backtracking step to the above cost Tables generated in Step 4:

The optimal tour is $1 \begin{array}{llllllllll}5 & 8 & 4 & 9 & 6 & 2 & 7 & 3 & 10 & 1\end{array}$

Use of Step 4:

If you got a tour already, (and tour length is (say OTL))

For example, if there are ' $N$ ' cities, and the number of cities that to visited already is ' $\boldsymbol{X}$ ' , and the distance travelled already is ' $\boldsymbol{D}$ ', Then it should travel atleast a distance of Minimum_Cost_Array[n-x+1] to get the next tour. So least possible distance for this tour is D + Minimum_Cost_Array[n-x+1] .

And it should satisy the condition " D + Minimum_Cost_Array[n-x+1] $<$ OTL " , then only it can say the path (tour) that are travelling now can be a minimum tour than the earlier tour OTL. If above condition is failed to satisfy then skip the combinations to improve efficiency.

But, in some cases if the number of edges removed are very less, that mean the cost table size is high then this algorithm won't give the optimal result, in that situation it is better to go for nearly optimal solution.

\subsection{Example:}

Consider this 24 city problem:

The Adjacency Matrix for this problem is shown on separate sheet in the next page.

But to calculate the optimal path from normal backtracking method need 23! combinations, tht is, 25852016738884976640000 combinations have to be performed, so that it go for the nearly optimal solution. This approach is efficient to find the nearly optimal solution.

The Optimal solution for the problem is

\section{2 $1 \quad$ Cost 1272}

But to get the solution in a polynomial time is not possible, so by giving time bound of 15 seconds to Edge Removal Algorithm, will get the nearly optimal cost of 1316 . This algorithm is checked against several samples of real world data and the results are promising to be nearly optimal. All the results shows this algorithm give the nearly optimal solution less than the 2 times of optimal solution in all possible cases. 
The adjacency matrix [2.2] :

\begin{tabular}{|c|c|c|c|c|c|c|c|c|c|c|c|c|c|c|c|c|c|c|c|c|c|c|c|}
\hline 0 & 57 & 187 & 91 & 50 & 80 & 130 & 134 & 243 & 185 & 214 & 70 & 272 & 219 & 293 & 54 & & 290 & 268 & 261 & 175 & 25 & 192 & 121 \\
\hline & 0 & 96 & 228 & 12 & 96 & 67 & 54 & 209 & 86 & 23 & 91 & 80 & 83 & 50 & 219 & 74 & 139 & 53 & 43 & 128 & & 228 & 142 \\
\hline & 196 & 0 & 58 & 6 & 88 & 59 & 63 & 86 & 124 & & & & & 232 & בר & & 98 & 138 & 200 & & & & 9 \\
\hline & 28 & 158 & 0 & 20 & 77 & 101 & 05 & & 156 & & 27 & & 49 & 264 & 02 & & 261 & 239 & 32 & 46 & & & \\
\hline & & 96 & 120 & 0 & 63 & 56 & 34 & 90 & 40 & & 83 & & 79 & 48 & & & & 123 & & 32 & & & 5 \\
\hline & & & & 63 & 0 & 25 & 29 & & & & & & & & & & & & & & & & \\
\hline & & 9 & 01 & 56 & 25 & 0 & 22 & & 95 & & 64 & & & 203 & 43 & & 64 & 178 & & & & & \\
\hline & 54 & 63 & 05 & 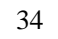 & 23 & 22 & 0 & 25 & 82 & 90 & & 28 & 12 & 190 & 8 & & 136 & 165 & 101 & 30 & 47 & 54 & 6 \\
\hline & 209 & 286 & 59 & 190 & & 229 & & 0 & 207 & & & & & & & & 89 & & & & & & \\
\hline & 86 & 124 & 56 & 40 & 124 & 95 & 82 & 207 & 0 & 51 & 10 & 150 & 62 & 122 & 147 & 37 & 110 & 86 & & 56 & & 136 & \\
\hline 214 & 225 & 49 & 85 & 123 & 115 & 86 & 90 & 313 & 151 & 0 & 40 & 342 & 199 & 259 & 04 & 100 & 141 & 101 & 227 & 103 & 138 & 262 & 120 \\
\hline 70 & 191 & 21 & 27 & 83 & 47 & 64 & & & 119 & & & 09 & 53 & & & 145 & 224 & 202 & 195 & 09 & & 10 & \\
\hline 272 & 180 & 315 & 188 & 193 & 245 & 258 & 228 & 29 & 159 & נד & 209 & 0 & 97 & 219 & 201 & 196 & 275 & 227 & 137 & 225 & & & 240 \\
\hline 13 & 83 & 172 & 49 & 79 & 139 & 134 & 112 & 126 & 6 & 99 & 53 & 97 & c & 134 & 10 & 99 & 178 & 130 & 69 & 104 & 38 & 96 & 04 \\
\hline 293 & 50 & 232 & 264 & 48 & 232 & 203 & 190 & 248 & 122 & 259 & 227 & 219 & 134 & ( & 255 & 125 & 154 & 68 & & 164 & 14 & 264 & 178 \\
\hline 54 & 219 & 92 & 82 & 119 & 31 & 42 & 58 & 238 & 147 & 84 & 35 & 267 & 170 & 255 & 0 & 173 & 190 & 230 & 223 & 99 & 212 & 101 & Do \\
\hline 211 & 74 & 81 & 182 & 105 & 50 & 121 & 108 & 310 & 3 & 60 & 45 & 196 & & 125 & 73 & & 79 & 5 & & & & 82 & 6 \\
\hline 290 & 139 & & 261 & 144 & 176 & 164 & 136 & 389 & 116 & & 224 & 275 & 178 & 154 & & 79 & & 86 & & & & & 75 \\
\hline 268 & 53 & 138 & 239 & 123 & 207 & 178 & 165 & 367 & 86 & 187 & 202 & 227 & 130 & 68 & 230 & 57 & 86 & 0 & 90 & 114 & 46 & 239 & . \\
\hline 261 & 43 & 200 & 232 & 98 & 200 & 171 & 131 & 166 & 90 & 227 & 105 & 137 & 69 & 82 & 223 & 90 & 176 & 90 & 0 & 134 & 136 & 165 & 146 \\
\hline 175 & 128 & 76 & 146 & 32 & 76 & 47 & 30 & 222 & 56 & 103 & 109 & 225 & 104 & 164 & 99 & 57 & 112 & 114 & & 0 & 96 & 151 & \\
\hline 250 & 99 & 89 & 221 & 105 & 189 & 160 & 147 & 349 & 76 & 138 & 184 & 235 & 138 & 114 & 212 & 39 & 40 & 46 & 136 & 96 & & 221 & 135 \\
\hline 172 & 228 & 235 & 108 & 119 & 165 & 178 & 154 & 71 & 136 & 202 & 100 & 74 & 96 & 264 & 187 & 182 & 261 & 239 & 165 & 151 & 221 & 0 & 103 \\
\hline & 142 & 99 & 84 & 35 & 29 & 42 & 36 & 220 & 70 & 126 & & 249 & 104 & 178 & 60 & 96 & 175 & & & 47 & & 169 & \\
\hline
\end{tabular}

The nearly optimal solutions found based on this algorithm within 15 seconds is given below:

\begin{tabular}{|c|c|}
\hline 166782152412419132314101719220152218311 & Cost 1509 \\
\hline 166782152412419132314101722015192218311 & Cost 1473 \\
\hline 166782152412419132314101720215192218311 & Cost 1457 \\
\hline 166782152412419132314202151910172218311 & Cost 1453 \\
\hline 166782152412142391314101722192201518311 & Cost 1443 \\
\hline 166782152412142391314101719220152218311 & Cost 1415 \\
\hline 166782152412142391314101722015192218311 & Cost 1379 \\
\hline 166782152412142391314101720215192218311 & Cost 1363 \\
\hline 166782152412142391314202151910172218311 & Cost 1359 \\
\hline 166782152410173111822192152014139234121 & Cost 1355 \\
\hline 166782152410173111822191522014139234121 & Cost 1331 \\
\hline 166782124510173111822191522014139234121 & Cost 1316 \\
\hline
\end{tabular}

\section{CONCLUSION}

This paper proposed a novel algorithm to get nearly optimal solution to the Travelling Sales man problem by taking time bound as a limit. This algorithm uses single source shortest path algorithm to remove unnecessary edges and by using compression algorithm. This algorithm is tested against several real world data and results are promising.

\section{ACKNOWLEDGEMENT}

Authors thanks to the management of Narasaraopeta Engineering College, for their continuous encouragement. Finally also thanks to the referees for their comments to improve the quality of this research paper.

\section{REFERENCES}

[1] A.V. Aho, J.E. Hopcroft and J.D. Ullman, "The Design and Analysis of Computer Algorithms", AddisonWesley, 1974.

[2] E. Horowitz and S. Sahni, "Fundamental of Computer Algorithms", Computer Science Press, 1982.

[3] E. L. Lawler, J. K. Lenstra, A. RinnooyKan, and D. B. Shmoys. The TravelingSalesman Problem: A Guided Tour of Combinatorial Optimization. Wiley,Chichester, England, 1985.

[4] Goldberg David E, Lingle R Jr. "Alleles, Loci, and the Traveling Salesman Problem." Proc. Of 1st Int. Conf. on Genetic Algorithms and Their Applications, Lawrence Erlbaum Associates, 1985,154-159 
[5] E. L. Lawler, J. K. Lenstra, A. RinnooyKan, and D. B. Shmoys. The Traveling Salesman Problem: A Guided Tour of Combinatorial Optimization. Wiley, Chichester, England, 1985.

[6] A. Gibbons and W. Rytter, "Efficient Parallel Algorithms" Cambridge University Press, 1988.

[7] M. Weiss, "Data Structures and Algorithm Analysis", Benjamin-Cummings, 1992

[8] U. Manber, "Introduction to Algorithms: A Creative approach”, Addison-Wesley, 1989.

[9] G. Gonnet and R. Baeza-Yates, "Handbook of Algorithms and Data Structures" , Addison- Wesley, 2 ed., 1991.

[10] B. Salzberg, "File Structures: An Analytic Approach", Prentice-Hall, 1988

[11] C. H. Papadimitriou and M. Yannakakis. The traveling salesman problem with distances one and two. Mathematics of Operations Research, 18(1):1-11, 1993.

[12] S. O. Krumke, W. E. de Paepe, D. Poensgen, and L. Stougie. News from the online traveling repairman. In J. Sgall, A. Pultr, and P. Kolman, editors, Proc. 26th Symp. on Mathematical Foundations of Computer Science, volume 2136 of Lecture Notes in Computer Science, pages 487-499. Springer-Verlag, 2001.

[13] Prof. Lenore Cowen, Scribe: Stephanie Tauber, Lecture notes on "The Travelling Salesman Problem (TSP)",
Comp260: Advanced Algorithms, Tufts University, Spring 2002.

[14] G. Gutin and A. P. Punnen, editors.The Traveling Salesman Problem and itsVariations. Kluwer, Dordrecht, TheNederlands, 2002.

[15] M. Lipmann. On-Line Routing.PhD thesis, Eindhoven University of Technology, 2003.

[16] K. Chaudhuri, B. Godfrey, S. Rao, and K. Talwar. Paths, trees, and minimum latency tours. In Proceedings of the 44th Annual Symposium on Foundations of Computer Science, Cambridge, Massachusetts, 2003.

[17] C. Chekuri and A. Kumar. Maximum Coverage Problem with Group Budget Constraints and Applications. Proc. Of APPROX-RANDOM, LNCS, 72-83, 2004.

[18] C. Chekuri and M. Pal. A Recursive Greedy Algorithm for Walks in Directed Graphs. Proc. of IEEE FOCS, 245-253, 2005.

[19] C. Chekuri and M. Pal. An O(log n) Approximation for the Asymmetric Traveling Salesman Path Problem. Proc. of APPROX, 95-103, 2005.

[20] K. Chen and S. Har-Peled. The Orienteering Problem in the Plane Revisited. Proc. of ACM SoCG, 247-254, 2006.

[21] V. Nagarajan and R. Ravi. Poly- logarithmic approximation algorithms for Directed Vehicle Routing Problems. Proc.of APPROX, 257-270, 2007. 